OPEN ACCESS

Edited by:

Brian C. Clark,

Ohio University, United States

Reviewed by:

David A. E. Bolton,

Utah State University, United States

Tibor Hortobagyi,

University Medical Center Groningen,

Netherlands

Edward Ofori,

Arizona State University,

United States

*Correspondence:

Jacqueline A. Palmer

palmerja1723@gmail.com

Received: 23 March 2021 Accepted: 17 June 2021

Published: 14 July 2021

Citation:

Palmer JA, Payne AM, Ting LH and Borich MR (2021) Cortical Engagement Metrics During Reactive Balance Are Associated With Distinct Aspects of Balance Behavior in

Older Adults.

Front. Aging Neurosci. 13:684743. doi: 10.3389/fnagi.2021.684743

\section{Cortical Engagement Metrics During Reactive Balance Are Associated With Distinct Aspects of Balance Behavior in Older Adults}

\author{
Jacqueline A. Palmer ${ }^{1 *}$, Aiden M. Payne ${ }^{1}$, Lena H. Ting ${ }^{1,2}$ and Michael R. Borich ${ }^{1}$ \\ 'Division of Physical Therapy, Department of Rehabilitation Medicine, Emory University, Atlanta, GA, United States, \\ ${ }^{2}$ Department of Biomedical Engineering, Emory and Georgia Tech, Atlanta, GA, United States
}

Heightened reliance on the cerebral cortex for postural stability with aging is wellknown, yet the cortical mechanisms for balance control, particularly in relation to balance function, remain unclear. Here we aimed to investigate motor cortical activity in relation to the level of balance challenge presented during reactive balance recovery and identify circuit-specific interactions between motor cortex and prefrontal or somatosensory regions in relation to metrics of balance function that predict fall risk. Using electroencephalography, we assessed motor cortical beta power, and beta coherence during balance reactions to perturbations in older adults. We found that individuals with greater motor cortical beta power evoked following standing balance perturbations demonstrated lower general clinical balance function. Individual older adults demonstrated a wide range of cortical responses during balance reactions at the same perturbation magnitude, showing no group-level change in prefrontal- or somatosensory-motor coherence in response to perturbations. However, older adults with the highest prefrontal-motor coherence during the post-perturbation, but not preperturbation, period showed greater cognitive dual-task interference (DTI) and elicited stepping reactions at lower perturbation magnitudes. Our results support motor cortical beta activity as a potential biomarker for individual level of balance challenge and implicate prefrontal-motor cortical networks in distinct aspects of balance control involving response inhibition of reactive stepping in older adults. Cortical network activity during balance may provide a neural target for precision-medicine efforts aimed at fall prevention with aging.

Keywords: aging - old age - seniors, dual task (DT), sensorimotor system, prefrontal cortex (PFC), functional connectivity, beta activity, response inhibition, posture

\section{INTRODUCTION}

The development of balance impairment with aging is common but poorly understood. The neural mechanisms by which some individuals maintain high levels of activity while others suffer a debilitating loss of mobility and independence remain elusive. During the aging process, there is a loss of automaticity in balance and mobility, where there 
may be a shift in whole-body postural control from a lower spinal and subcortical level (Kim et al., 2013) to a higher brain center mediation (Alizadehsaravi et al., 2020). However, a consequence of this neuromechanistic shift is that engagement of cortical resources for balance control may interfere with older adults' ability to perform cognitive and mobility tasks simultaneously (Lundin-Olsson et al., 1997; Jacobs and Horak, 2007; Maki and McIlroy, 2007). Previous studies have identified active cortical regions during continuous balance and walking tasks in older adults (Chang et al., 2016; Malcolm et al., 2020), but cortical oscillatory activity time-locked to destabilizing balance events, shown to be associated with balance ability in younger adults (Ghosn et al., 2020), or interactions between cortical regions reflecting information processing and integration during motor behavior have not been well investigated in older adults, who, as a group, have a higher risk for falling. Such knowledge could identify effective neural control strategies during balancecorrecting behavior in high-functioning older adults and could be leveraged towards the development of precision medicine approaches for individualized fall prevention strategies among a heterogeneous older adult population. In the present study, we used electroencephalography (EEG) to measure time-locked cortical activity during standing balance reactions in a group of older adults across a range of individual balance abilities. We aimed to characterize the neural dynamics of cortical oscillatory activity and interactions between cortical regions during balance reactions, and test the relationship between individual cortical engagement strategy during balance reactions and distinct aspects of balance behavior that are predictive of falls in older adults.

Balance control is multifactorial. A variety of tests measure different aspects of balance control, whose neural underpinnings are not well understood. Growing evidence shows that engagement of cortical resources during balance is an indicator of fall risk in older adults, where a concurrent cognitive task shifts cortical resources away from balance control (LundinOlsson et al., 1997; Shumway-Cook et al., 1997; Woollacott and Shumway-Cook, 2002; Montero-Odasso et al., 2012). Commonly used clinical tests, such as the miniBEST, that assess a myriad of aspects of postural control including single-task, cognitive dual-task, and reactive balance, have high clinical utility, but are nonspecific, lack precision, and can impose a ceiling effect on individuals with higher balance ability (Marques et al., 2016). The ability to react to a loss of balance is a key factor that ultimately determines whether an individual will sustain a fall. These balance recovery mechanisms can be profoundly impaired in older adult populations (for a comprehensive review see Maki and McIlroy, 1996, 2006) and are strongly linked to fall risk (Wolfson et al., 1986; Chandler et al., 1990). The link between balance recovery ability and fall risk has prompted researchers to quantify reactive balance capacity as the initiation of stepping responses during postural destabilization of a given magnitude, where individuals with greater reactive balance impairment require stepping reactions at lower levels of balance perturbations (Jensen et al., 2001; Mille et al., 2003). Reactive balance recovery also provides a unique paradigm to assess cortical activity dynamics that are time-locked to balance behavior using electroencephalography (EEG). In the present study, we aimed to quantify individual reactive balance capacity by increasing the magnitude of balance perturbations to the point where balance challenge exceeded the capacity of an individual to produce feet-in-place reactions, necessitating a later-phase reactive stepping response that is likely cortically-mediated (Maki and McIlroy, 2007). Previously, our lab used EEG to assess cortical activity during balance reactions in younger adults and found larger evoked cortical responses in individuals with lower balance performance on a beam walking task (Ghosn et al., 2020; Payne and Ting, 2020). Further, evoked cortical activity was dissociable from evoked muscle activity, suggesting a potential cortical motor contribution to later-phase reactive balance control (Payne et al., 2019a). However, it is unclear whether older adults engage similar cortical strategies during balance reactions, and whether behavioral assessments of fall risk used in older adult populations assess similar or distinct aspects of balance control. Moreover, understanding the neural control strategies underpinning the various aspects of balance would be useful for the development of fall prevention treatments within a precisionmedicine framework.

Investigating the information processing between motor cortical and other brain regions during whole-body balancecorrecting behavior could provide valuable information about the time course of circuit-specific cortical contributions to balance control that cannot be observed using measures of cortical activity during rest or static balance alone. Neural oscillations in the beta frequency band $(13-30 \mathrm{~Hz})$ are a prominent feature of motor behavior (Engel and Fries, 2010; van Wijk et al., 2012; Zaepffel et al., 2013). In older adults, and in age-related neurodegenerative diseases such as Parkinson's disease, abnormal movement-related beta oscillatory modulation has been associated with slowed and impaired volitional motor activity (Brown, 2007; Johari and Behroozmand, 2020). Consistent with age-related increases in cortical recruitment during motor tasks (Seidler et al., 2010), older adults had greater movement-related modulation of beta activity compared to younger adults during volitional manual movements (Rossiter et al., 2014). Age-related differences in functional connectivity between cortical regions may, in part, explain differences in movement-related cortical beta activity in older adults, which has been observed in older individuals with Parkinson's disease and stroke (Rowe et al., 2002; Grefkes et al., 2008). Functional connectivity analyses performed during resting motor states suggest that the role of neural interactions between cortical regions may be circuitspecific (Langan et al., 2010; Solesio-Jofre et al., 2014; Seidler et al., 2015). However, the functional role of cortical beta oscillations and circuit-specific functional connectivity in the aging brain remains less clear in behavioral contexts, particularly whole-body balance reactions.

Heightened cortical activity in somatosensory and motor regions has been observed in older adults compared to younger individuals and associated with upper limb motor function (Mattay et al., 2002; Heuninckx et al., 2008; Cassady et al., 2020), but it is unclear whether the sensorimotor cortical activity 
is also associated with balance function. Centrally-mediated sensorimotor processing within the cortex may preserve balance control in the presence of age-related loss of somatosensory function (Zhang et al., 2011) and the loss of automaticity of balance and mobility behavior via subcortical mechanisms (Clark, 2015). Older adults consistently show a greater extent of activation in somatosensory and motor cortical brain regions during a myriad of single-segment limb motor tasks or tasks that mimic whole-body task performance (e.g., virtual reality or mental imagery) compared to younger adults (Mattay et al., 2002; Heuninckx et al., 2008; Goble et al., 2012; Zwergal et al., 2012; Cassady et al., 2020). Increases in somatosensory and motor cortical activity with aging have also been accompanied by greater functional connectivity between somatosensory and motor regions during finger tapping tasks, suggesting causal network interactions between these brain regions (Cassady et al., 2020). Though cortical interactions between somatosensory and motor regions have been historically challenging to study in the context of whole-body behaviors, higher levels of sensorimotor cortical activity have been positively associated with interlimb coordination performance (Heuninckx et al., 2008), potentially implicating a beneficial functional role for sensorimotor cortical control in bilateral limb motor performance during standing balance and mobility. In this study, we sought to test somatosensory-motor interactions during reactive balance and its association with balance ability in older adults.

Prefrontal cortical brain regions subserving cognitive executive function and working memory appear to play an increased functional role in balance control with aging and may limit simultaneous cognitive/balance task performance and the upper limit of individual motor performance capacity in older adults. Cognitive interference during balance and mobility in older adults (Brown et al., 1999; Rankin et al., 2000; Woollacott and Shumway-Cook, 2002; Morris et al., 2016; Leone et al., 2017) suggests the development of overlapping cortical control mechanisms for cognitive and motor control processes with aging (Ren et al., 2013; Cid-Fernández et al., 2014), implicating an increased role of the prefrontal cortex for balance control. Older adults show greater prefrontal cortical activity during a wide range of motor tasks, including interlimb coordination tasks (Heuninckx et al., 2008) and steady-state walking (Chen et al., 2017; Mirelman et al., 2017; Hawkins et al., 2018). Whether age-related differences in prefrontal cortical activity play a beneficial (i.e., compensatory; Clark et al., 2019) or detrimental (i.e., age-related neural dedifferentiation; Payer et al., 2006; Gagnon et al., 2019) role in motor function in older adults remains controversial (for review see Seidler et al., 2010) and may depend on the context and challenge of the motor task (e.g., level of complexity and difficulty; Clark, 2015). In contrast to younger adults, older adults utilize motor control strategies that require higher levels of cognitive processing, which are effective at slower speeds but less effective during fast speed motor performance (Boisgontier and Nougier, 2013). Additionally, individuals who show greater prefrontal cortex activity at low levels of task difficulty appear to have limited ability for additional prefrontal resource recruitment as the complexity and challenge of the task increases, ultimately limiting the upper end of performance capacity (Hawkins et al., 2018). In this study, we sought to test whether the level of engagement of prefrontal-motor cortical networks during balance reactions at the same perturbation magnitude across participants was associated with the level of cognitive dual-task interference (DTI) and reactive balance performance under challenging perturbation conditions.

In the present study, we aimed to investigate motor cortical beta activity and circuit-specific interactions between motor and prefrontal or somatosensory cortical brain regions over the time course of standing balance recovery in older adults. We tested the relationship between motor cortical beta power and beta coherence of EEG electrodes overlying frontal and central brain regions with three key aspects of functional balance ability in older adults, namely general clinical balance function, cognitive dual-task interference, and upper-end reactive balance capacity measured as reactive step threshold. We hypothesized that somatosensory-motor cortical networks contribute to balance control in older adults and therefore greater somatosensorymotor coherence would be present in older adults with higher balance ability. We predicted that individuals with greater recruitment of prefrontal-motor circuits at the same perturbation magnitude would be more susceptible to cognitive dual-task interference, showing more slowing in their mobility performance during simultaneous cognitive task performance, and have lower reactive balance capacity, measured as eliciting of a stepping reaction at lower perturbations as magnitude was increased.

\section{MATERIALS AND METHODS}

\section{Participants}

Sixteen individuals were recruited from the local Atlanta community to participate in this study. All participants completed a single testing session consisting of clinical balance assessments and neurophysiologic testing during a standing balance perturbation series. Inclusion criteria included above the age of 50, the ability to walk at least 10 meters without the assistance of another person, the ability to stand unassisted for at least $3 \mathrm{~min}$, and the cognitive ability for informed consent. No participants in the present study used an assistive device for ambulation. Participants were excluded if they had been diagnosed with any neurologic condition, any musculoskeletal condition that affected their standing or walking, peripheral neuropathy, or pain affecting standing or walking. The experimental protocol was approved by the Emory University Institutional Review Board and all participants provided written informed consent.

\section{Behavioral Balance Assessments General Clinical Balance Function}

Upon arrival to the lab and prior to neurophysiologic instrumentation, participants completed the miniBEST to assess general clinical balance function. The miniBEST is a validated and commonly used clinical assessment for assessing static and dynamic balance ability and fall risk in elderly adults (Marques et al., 2016). Briefly, the miniBEST assesses domains 
of anticipatory balance control, reactive postural control, sensory orientation, and dynamic gait using a likert subscale of $0-2$ for each domain, where higher scores indicate better performance. The total sum of the itemized subscale scores represented miniBEST total score, with a maximum possible score of 28 (taking the lower of two scores for items scored separately for left and right legs).

\section{Dual-Task Interference}

Dual-task interference was assessed during a clinical TimedUp-and-Go (TUG) test, a validated clinical test for fall risk assessment in elderly populations (Tang et al., 2015). Participants started in a seated position with their back against the back of a chair. When the clinician verbally cued a "Go" signal, the participant stood up from the chair, walked 3 meters until both feet crossed over a taped line on the floor, and walked back to the chair, with the time stopping when their back came in contact with the back of the chair. Next, the participants were instructed to repeat the TUG test while performing the secondary cognitive task of verbally counting backward by 3's starting at a random integer number between 20 and 100 verbally stated by the experimenter immediately following the "Go" signal. Participants were instructed that this was a timed test and to "walk as fast as you safely can" during both single and dual-task performance. DTI was quantified as Plummer and Eskes (2015).

$$
\operatorname{DTI}(\%)=-\frac{(\text { dual task time }- \text { single task time })}{(\text { single task time })} * 100 \%
$$

negative DTI values indicate slower TUG performance during dual-task relative to the single-task condition. All clinical testing was administered by a licensed physical therapist.

\section{Behavioral Reactive Balance Capacity}

We identified step threshold as a method to quantify the level of balance challenge posed to each individual. We assessed individual behavioral reactive balance capacity by determining the lowest perturbation magnitude which elicited unintentional stepping reactions in approximately $50 \%$ of the trials, defined as the step threshold. The step threshold testing was performed after a seated rest break following the first series of perturbations to avoid initial behavioral adaptation effects that could occur at the start of the moving platform series. Participants stood on the platform with the same instructions to attempt to respond with a feet-in-place strategy and arms crossed in place at the chest. Forward direction perturbations were delivered starting at a magnitude of $8 \mathrm{~cm}$ with a jittered inter- trial interval of 15-60 s. To reduce anticipation of perturbation direction, backwards directional perturbations were also randomly administered with this perturbation series. If the participant successfully completed three consecutive feet-inplace trials, then the perturbation magnitude was scaled up by $1 \mathrm{~cm}$ displacement, with proportional increases in perturbation velocity and acceleration. This procedure was repeated, scaling up the perturbation magnitude until a step reaction occurred. At that point, the perturbation magnitude was held constant at this level for the next 10 perturbations. If the participant elicited five step reactions in a row at this perturbation magnitude, the perturbation magnitude was scaled down by 0.5 magnitude level. The step threshold was defined as the perturbation magnitude at which the participant utilized an unintentional step response strategy in approximately 5 out of $10(50 \%)$ trials. During this perturbation series, the experimenter closely monitored the participant's real-time force data during baseline quiet standing to ensure the same baseline standing position. If the participant attempted to adjust their posture in anticipation of a perturbation (e.g., increase stance width or forward trunk lean), the experimenter cued the participant to return to their normal baseline standing posture prior to perturbation delivery.

\section{Balance Perturbations}

\section{Standardized Perturbation Level Protocol}

To test for the effect of individual differences in balance challenge on cortical engagement, the same perturbation was delivered to all participants. Participants stood with bare feet in the middle of a moveable custom platform (Factory Automation Systems, Atlanta, GA) while support-surface translational perturbations were delivered in an unpredictable direction and at unpredictable timing. Twenty-four perturbations of equal magnitude $(7.5 \mathrm{~cm}$, $16.0 \mathrm{~cm} / \mathrm{s}, 0.12 \mathrm{~g}$ ) were delivered in the forward direction to elicit a backwards center of mass displacement relative to the base of support. The scaling of these perturbation parameters was selected to ensure that platform deceleration did not occur until $500 \mathrm{~ms}$ after perturbation onset to minimize changes in cortical and motor output originating from a deceleration response (McIlroy and Maki, 1994; Ghosn et al., 2020). Because we aimed for each participant to sustain identical perturbations, we selected this lower-level perturbation magnitude as a level of postural destabilization that could be successfully completed by most older adults using a feet-in-place strategy (Figure 1). To reduce the directional anticipation and time of perturbation onset, we included three additional perturbation directions (backwards, 45 degrees right posterolateral, 45 degrees left posterolateral) using the same scaled parameters into the perturbation series in a pseudorandomized order, where a perturbation of the same magnitude had no more than two consecutive occurrences. To reduce anticipation of precise perturbation onset, perturbations were delivered at a jittered inter-trial interval, with $15-60 \mathrm{~s}$ between each perturbation onset. Real-time EEG activity and force feedback was also monitored by the experimenter to ensure that the participant returned to baseline levels of cortical and muscle activity and maintained the same baseline body position. Participants were asked to attempt to recover balance while maintaining both feet in place and arms crossed at their chest. If a participant executed an unintentional stepping reaction in response to a perturbation by visual determination by the experimenter in real time, the trial was marked for offline confirmation and exclusion based on ground reaction forces. Participants took a seated rest break every 8 min during balance perturbation testing, or more frequently if the participant 


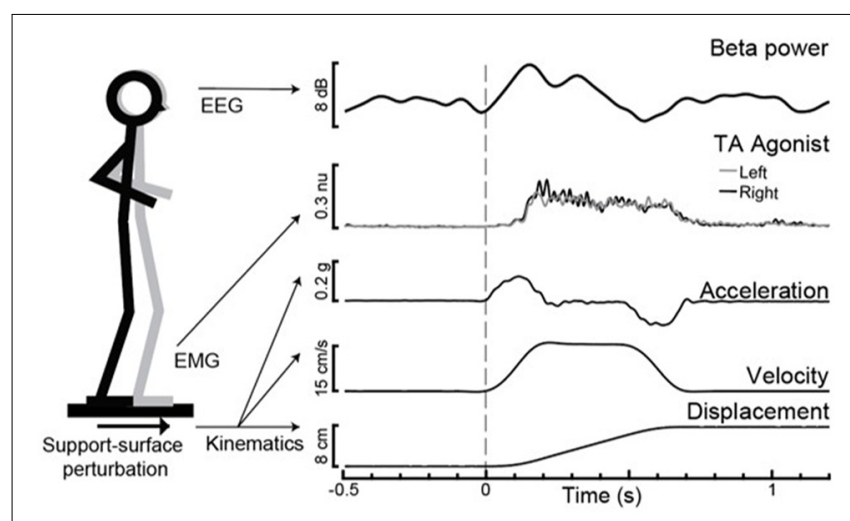

FIGURE 1 | Experimental paradigm with evoked motor cortical beta power $(\mathrm{Cz})$ and tibialis anterior (TA) agonist muscle activity with support-surface perturbation kinematics for an exemplar participant.

requested a break or reported or showed signs of fatigue during testing.

\section{Electroencephalography (EEG) Data Collection and Analyses}

During the standardized-level balance perturbations, EEG signals using a 64-channel active electrode cap (ActiCap, Brain Products $\mathrm{GmbH}$, Gilching, Germany) connected to an ActiCHamp amplifier (Brain Products, $\mathrm{GmbH}$ ). Data were continuously recorded and online referenced to the Fz channel (Recorder, Brain Products, $\mathrm{GmbH}$ ). EEG signals were digitized with a 24-bit analog-to-digital converter and an online $20 \mathrm{kHz}$ low-pass filter and were sampled at $1,000 \mathrm{~Hz}$ and stored for offline analyses.

\section{Preprocessing}

All EEG data were preprocessed using freely available functions from the EEGlab toolbox (Delorme and Makeig, 2004). The time-locked continuous data were imported into EEGlab and filtered with a high-pass cutoff of $1 \mathrm{~Hz}$ and a low-pass cutoff of $100 \mathrm{~Hz}$. Next, the events with trigger labels for successful trials (no step) in the forward direction of platform translation were selected $(-2$ to $3 \mathrm{~s}$ relative to the time of the platform movement onset trigger at $t=0$ ) and any trial that was contaminated by artifacts was removed from the analysis. Highly contaminated channels outside of the channels used for primary analyses $(\mathrm{Cz}, \mathrm{CPz}, \mathrm{AFz})$ were identified by visual inspection and removed from the recordings. On average, 62 of 65 channels remained for analyses ( $\mathrm{SD} \pm 3.4$ ); range 53-65. The removed electrodes were then interpolated using the pop_interp function in EEGlab. The Cleanline plugin for EEGLAB was applied to the continuous data to remove line noise $(60 \mathrm{~Hz})$. Next, the data were epoched $(-1$ to $2 \mathrm{~s}$ relative to perturbation onset). We then applied Independent Component Analysis (ICA) to remove non-neural artifacts of the EEG signal (e.g., muscle activity, motion, eyeblinks). Using as few as 32 electrodes, ICA has been shown to effectively disentangle motor, sensory, and cognitive processes, even when they are occurring simultaneously with overlapping scalp distributions and frequency properties (Makeig et al., 2004). Non-neural artifacts (e.g., muscle activity, motion artifact, eye blinks, cardiac rhythm) were identified using an automatic component selection TESA algorithm (Rogasch et al., 2017) and visually confirmed for accuracy. The remaining components were retained for subsequent analyses.

\section{Quantification of Cortical Beta Power}

Time-frequency decomposition analyses were used to quantify changes in beta oscillatory power in response to balance perturbations for the vertex electrode overlying the primary motor cortex $(\mathrm{Cz})$. We used the wavelet time-frequency analyses function (pop_newtimef.m) in EEGLAB to quantify beta oscillatory power across all forward perturbations within each participant. We used a sliding window of $256 \mathrm{~ms}$ to measure power at each frequency using a tapered Morlet wavelet. The lowest frequency $(12 \mathrm{~Hz})$ used three oscillatory cycles, which increased up to six cycles used at the highest frequency $(50 \mathrm{~Hz})$. The event-related spectral perturbation (ERSP) quantified oscillatory power (Makeig, 1993) at 10 linearly spaced frequencies $(12 \mathrm{~Hz}$ to $50 \mathrm{~Hz}$ ) at $14 \mathrm{~ms}$ intervals throughout the perturbation trials. To index power in the beta frequency domain, the mean of the ERSP values across four sampled frequencies centered on $16 \mathrm{~Hz}, 20 \mathrm{~Hz}, 24 \mathrm{~Hz}$, and $29 \mathrm{~Hz}$ were computed for each participant. We then computed the mean baseline beta power $(-500$ to $0 \mathrm{~ms}$ before perturbation onset) and the peak of the perturbationevoked change in beta power (100-500 ms) relative to baseline for each participant. Based on our previous study which found differences between early and later portions of motor cortical beta activity responses as a function of balance ability in younger adults (Ghosn et al., 2020), we further identified peak perturbation-evoked beta power within an early (100-300 ms) and later (300-500 ms) time window of the response.

\section{Quantification of Circuit-Specific Cortical Coherence}

The imaginary part of coherence (IPC) analyses (Nolte et al., 2004) were used to quantify the phase-shifted synchrony of oscillatory activity within the beta frequency range $(13-30 \mathrm{~Hz})$ between two pairs of vertex electrodes approximately overlying lower limb regions of the primary motor cortex $(\mathrm{Cz})$ with: (1) primary somatosensory $(\mathrm{CPz})$ and; (2) prefrontal cortical $(\mathrm{AFz})$ regions. Given the close anatomical proximity of our brain regions of interest, we chose this methodologically conservative coherence analysis approach to minimize the risk of artificially inflated cortical coherence due to volume conduction by requiring a phase lag between distinct source signals (Nolte et al., 2004). Analyses were performed using custom routines in MATLAB, using an upper-end frequency cut-off of $50 \mathrm{~Hz}$, segment length of 768, and overlay of 0.9 (Palmer et al., 2020) to yield a frequency domain resolution of $1.3 \mathrm{~Hz}$ and time domain resolution of $76.8 \mathrm{~ms}$. The pre-perturbation IPC value within the $400 \mathrm{~ms}$ prior to perturbation onset ( -400 to $0 \mathrm{~ms}$ ) was computed from the mean of the six segments prior to $t=0$, with the first segment centered at $-388 \mathrm{~ms}$ and the last segment at $-8 \mathrm{~ms}$. The time window of 100-500 ms post-perturbation onset was selected 
because it captured the earliest occurrence of motor cortical beta power change within the group (Figure 2) and would not be affected by the platform deceleration after $500 \mathrm{~ms}$ (Figure 1). The post-perturbation (100-500 ms) IPC value was computed from the mean of the first five segments relative to $t=0$, with the first segment centered at $144 \mathrm{~ms}$ and the last segment at $448 \mathrm{~ms}$.

\section{Statistical Analyses}

We used Kolmogorov-Smirnov and Levene's tests to test for normality and homogeneity of variance in balance behavior, cortical beta power, and cortical beta coherence data. We tested the relationship between clinical balance behavioral measures of miniBEST, dual-task interference, and reactive step threshold using Pearson product-moment correlation coefficients. We tested the modulation of motor cortical beta power, somatosensory-motor beta coherence, and prefrontalmotor beta coherence between pre- and post-perturbation time windows during balance reactions using paired t-tests. We tested the relationship between perturbation-evoked motor cortical beta power and miniBEST score using Pearson product-moment correlation coefficients. We used Pearson product-moment correlation coefficients with False Discovery Rate (FDR) adjusted p-values to test relationships between pre- and post-perturbation somatosensory-motor and prefrontal-motor beta coherence vs. miniBEST, dual-task interference, and step threshold. Statistical analyses were performed using Statistical Package for Social Sciences version 27 (IBM Corp, Armonk, NY) with an a priori $\alpha$ level set to 0.05 .

\section{RESULTS}

In summary, we analyzed a mean of 22 trials for 14 participants, with one participant completing the protocol at a reduced perturbation magnitude and one participant including backward in addition to forward directional perturbations. Fifteen (age: $69 \pm 8$ years, 11 female, Table 1 ) out of 16 participants were able to complete the protocol. One participant withdrew from the study due to high levels of anxiety and fear of falling during platform movement and was excluded from all analyses. One participant was only able to successfully execute four trials in the forward direction without a stepping reaction; beta coherence showed no difference between forward and backward directions, and thus successful no step trials in the backward direction were included in EEG analysis for this participant to standardize the number of trials across participants. One other participant was unable to successfully respond to balance perturbations at the $7.5 \mathrm{~cm}$ magnitude with feet in place in any direction; for this participant, the perturbation parameters were scaled down to $7 \mathrm{~cm}$ magnitude displacement $(7 \mathrm{~cm}$, $15 \mathrm{~cm} / \mathrm{s}, 0.12 \mathrm{~g}$ ), a level where feet-in-place trials could be successfully executed. As a group, participants were able to successfully recover balance without stepping in $91.3 \pm 1.4 \%$ of the trials on average. Thus, an average of $21.9 \pm 5.0$ trials was used in the final EEG data analyses across participants. The EEG recordings of one other participant had excessively high impedances $(>50 \mathrm{kOhm})$ secondary to the use of an oil-based hair product on the scalp prior to testing; these data were excluded from EEG analyses but retained for balance behavioral analyses.

\section{Assessment of Clinical Balance Function and Reactive Balance Capacity}

When testing the relationship between behavioral balance measures, we found a strong relationship between cognitive dual-task interference and reactive step threshold, while there was no significant relationship between general clinical balance function, measured as the miniBEST, and either cognitive dual-task interference or reactive step threshold. Individual miniBEST scores were not associated with cognitive DTI $\left(r_{(15)}=0.38, p=0.16\right.$; Figure $\left.2 \mathrm{~A}\right)$ or reactive step thresholds $\left(r_{(15)}=0.47, p=0.07\right.$; Figure 2B). Individual cognitive dual-task interference was positively associated with reactive step threshold $\left(r_{(15)}=0.76, p=0.001\right)$, where individuals with greater slowing during the cognitive dual-task condition elicited reactive stepping responses at lower perturbation magnitudes (Figure 2C).
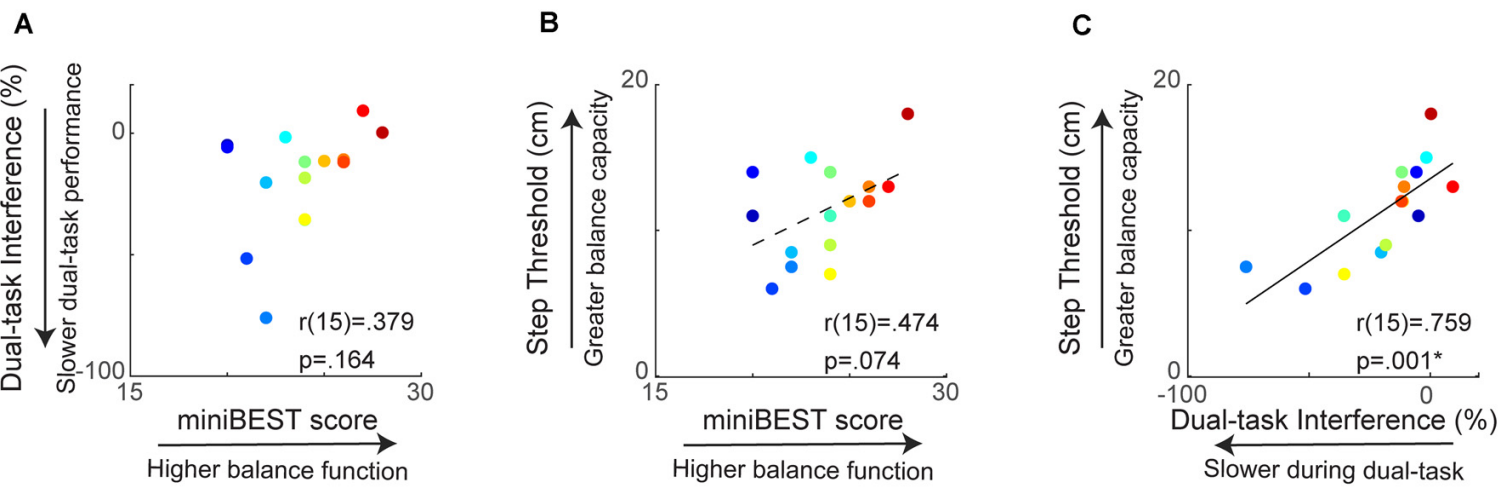

FIGURE 2 | Relationship between clinical behavioral balance function and reactive step threshold. MiniBEST score was not significantly associated with cognitive dual-task interference (DTI) (A) or reactive step threshold (B). Cognitive DTI was positively correlated with reactive step threshold, where older adults with more slowing during dual-task performance had lower reactive step thresholds $\left({ }^{*} p=0.001\right)$ (C). 
TABLE 1 | Participant characteristics.

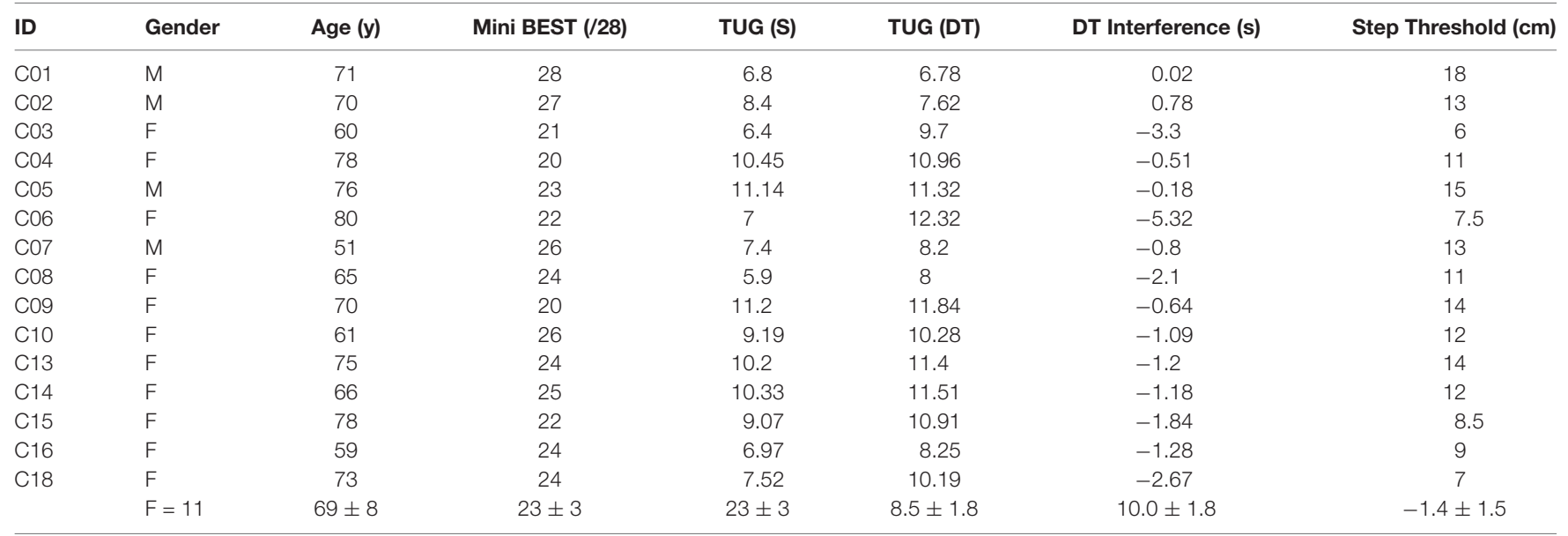

s: seconds.

\section{Perturbation-Evoked Motor Cortical Beta Power}

We found that older adults with greater later-phase perturbationevoked motor cortical beta power had lower levels of general clinical balance function, as measured by the miniBEST. Balance perturbations elicited an increase in motor cortical beta oscillatory power from the baseline mean $(27 \pm 2 \mathrm{~dB})$ to a post-perturbation (100-500 ms) mean ( $35 \pm 5 \mathrm{~dB} ; t=12.25$, $p<0.0,001$; Figure 3). There was no relationship between perturbation-evoked beta power during the overall (100-500 ms; $\left.r_{(14)}=-0.48, p=0.08\right)$, or early-phase $\left(100-300 \mathrm{~ms} ; r_{(14)}=-0.49\right.$, $p=0.07$ ) time window, and miniBEST (Figures $4 \mathbf{A}, \mathbf{B}$ ). Laterphase perturbation-evoked beta power was inversely associated with miniBEST $\left(r_{(14)}=-0.56, p=0.04\right)$, where individuals with higher perturbation-evoked beta power during the laterphase of balance reactions had lower clinical balance function (Figure 4C). We did not observe an association between perturbation-evoked beta power during any time window and cognitive dual-task interference or reactive step threshold $(p>0.05)$.

\section{Perturbation-Evoked Motor Cortical Coherence}

Older adults who showed greater prefrontal-motor coherence had greater levels of cognitive dual-task interference. Though balance perturbations did not elicit a change in somatosensorymotor [pre-(0.061 \pm 0.025$)$ to post- $(0.063 \pm 0.024 ; t=-0.55$, $p=0.59)]$ or prefrontal-motor [pre- $(0.125 \pm 0.052)$ to post(0.115 $\pm 0.038 ; t=1.47, p=0.17)]$ beta coherence at the group level, responses were highly variable between individuals, particularly between those with higher vs. lower balance behavioral scores (Figure 5A). There was no relationship between somatosensory-motor coherence and any clinical or behavioral balance behavioral measure (Table 2). While there was no relationship between prefrontal-motor coherence and miniBEST (Table 2), we observed a negative relationship between post-perturbation prefrontal-motor coherence and both reactive step threshold $\left(r_{(14)}=-0.66, p=0.03\right.$; Figure 5B) and cognitive dual-task interference $\left(r_{(14)}=-0.61, p=0.03\right.$; Figure 5C), where older adults with greater prefrontalmotor beta coherence elicited stepping reactions at smaller perturbation magnitudes and showed greater performance decline with cognitive loading. This relationship was not present at the pre-perturbation time point [dual-task interference $\left(r_{(14)}=-0.42, p=0.12\right)$; step threshold $\left(r_{(14)}=-0.43\right.$, $p=0.10)]$.

\section{DISCUSSION}

Our results provide an individualized framework for understanding cortical contributions to balance control, suggesting circuit-specific compensatory roles of cortical engagement in balance control. A key novel finding of the present study is that greater cortical engagement during balance reactions was present in older adults with lower general clinical balance ability and that interactions between prefrontal and motor cortical regions were associated with distinct aspects of balance behavior. The current findings shed light on individual-specific neural network dynamics during balance that can be gleaned from noninvasive electrophysiologic scalp recordings and using a simple and minimal electrode-based analysis approach, facilitating possible clinical translation as a biomarker in aging patient populations. Our results yield three main findings: (1) Similar to our previous findings in younger adults (Ghosn et al., 2020), perturbation-evoked beta oscillatory activity over central midline motor cortical regions was negatively correlated with balance ability in older adults, suggesting that perturbation-evoked cortical beta activity may provide a biomarker of individual level of balance challenge across the lifespan; (2) Individual older adults demonstrate a wide range of cortical engagement levels involving prefrontaland somatosensory-motor circuits during balance behavior; and (3) Greater perturbation-evoked prefrontal-motor cortical network connectivity was associated with greater decline in 


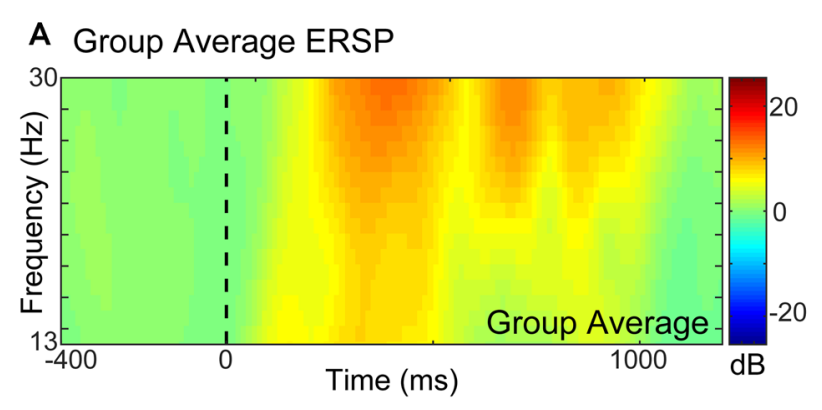

B Example Participant with Higher Balance Ability

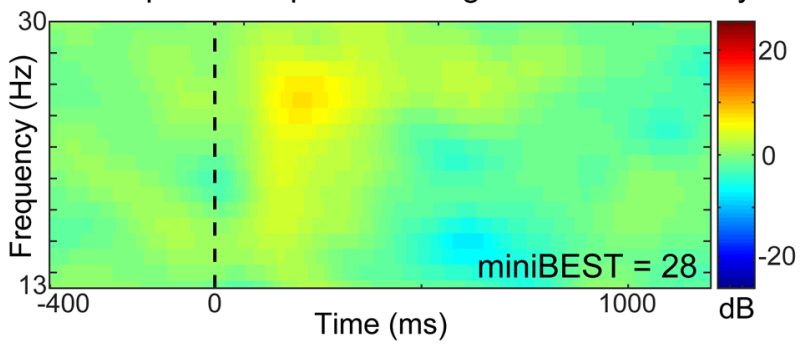

C Example Participant with Lower Balance Ability

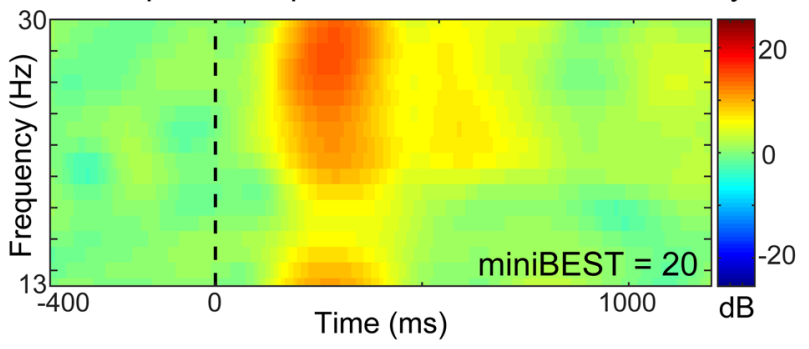

\section{Group and Individual Beta Power}

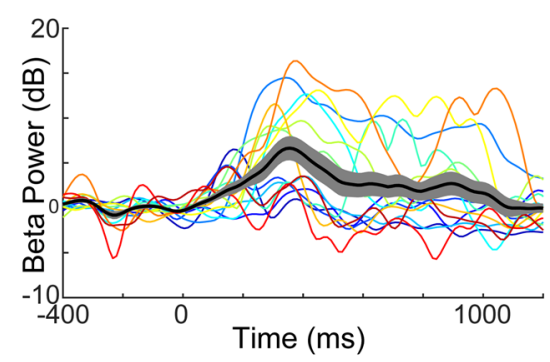

\section{E Peak Beta Power}

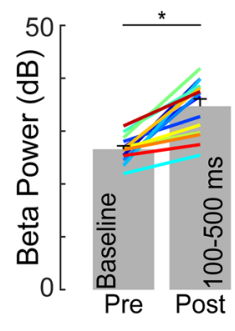

FIGURE 3 | Motor cortical beta oscillatory power $(\mathrm{Cz})$ during reactive balance responses. Group level event-related spectral perturbation (ERSP) in the beta frequency range $(13-30 \mathrm{~Hz})$. (A) ERSP in two exemplary individuals with higher (B) and lower (C) miniBEST score. Time course of beta power response across individuals. (D) Beta power increased from pre-(27 $\pm 2 \mathrm{~dB})$ to post-perturbation (100-500 ms; $35 \pm 5 \mathrm{~dB}$; $\left.{ }^{\star} p<0.0001\right)$ (E).

balance performance during cognitive loading and a lower threshold for eliciting stepping reactions, suggesting prefrontalmotor cortical circuits may mediate individual reactive balance capacity in older adults. Together, these findings provide evidence for feedback-driven individual strategies for cortical engagement that interact with factors within the environment in a context-dependent manner to influence balance behavior. Further, during balance recovery behavior involving inhibition of stepping responses, we identify key differences in cortical mechanisms involving top-down inhibition between older adults of different balance abilities.

\section{Cortical Beta Power as a Biomarker for Individual Balance Challenge}

Our findings implicate perturbation-evoked motor cortical beta power as a biomarker for individual balance challenge in older adults. We found that older adults with greater perturbationevoked beta power had lower general clinical balance ability, measured with the miniBEST (Figures 3, 4). These results are consistent with our previous study in younger adults, who underwent perturbations of larger magnitudes (Ghosn et al., 2020). We specifically observed this relationship in evoked cortical beta power during the later-phase (300-500 ms) of the reactive balance response (Figure $4 \mathrm{C}$ ), consistent with the timing of potential later-phase cortical contributions to reactive balance control (Rankin et al., 2000; Maki and McIlroy, 2007) and also in agreement with younger adults in our previous study (Ghosn et al., 2020). Thus, increased recruitment of cortical resources as the level of balance difficulty increases appears to be a strategy utilized by neurotypical younger adults, even in the absence of balance impairment on standard clinical tests (Ghosn et al., 2020). Together with the findings of the present study, our results suggest that cortical beta power may reflect a global upregulation of cortical engagement when the balance is challenged within an individual across the lifespan. Determination of individual level of balance challenge has important implications for precision medicine approaches to balance treatments and interventions. Motor task practice at precise levels of challenge is a necessary condition to maximize functional cortical neuroplasticity, particularly in older adult populations where, in contrast to young individuals, task practice at the highest levels of motor task challenge can impede skill retention (Bootsma et al., 2020). Thus, the utilization of motor cortical beta activity as a biomarker of individual level of balance challenge (Guadagnoli and Lee, 2004) could serve as a useful clinical tool to tailor individualized treatments and improve balance rehabilitation outcomes for fall prevention. For example, clinicians could use cortical biomarkers to reveal the neural control strategy an individual engages during balance tasks, identifying preclinical older adults at the earliest stages of balance impairments prior to falls, and who could be targeted for subsequent intervention for fall prevention. The association between metrics of brain activity measured during a standing reactive balance paradigm with dynamic balance ability and performance in the present study and others (Ghosn et al., 2020) implicate similar neural control mechanisms are involved in the control of standing balance reactions and dynamic balance behavior. These findings motivate future studies to investigate whether similar measures of brain activity are evoked by balance recovery during dynamic mobility behaviors such as walking, the behavioral context during which older adults most commonly experience a fall (Li et al., 2006; Robinovitch et al., 2013). 
A

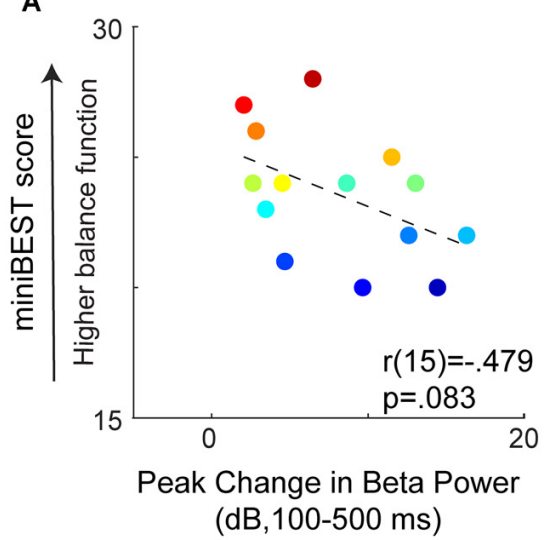

B

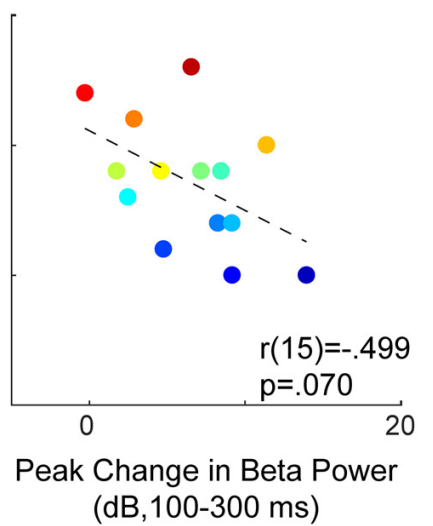

c

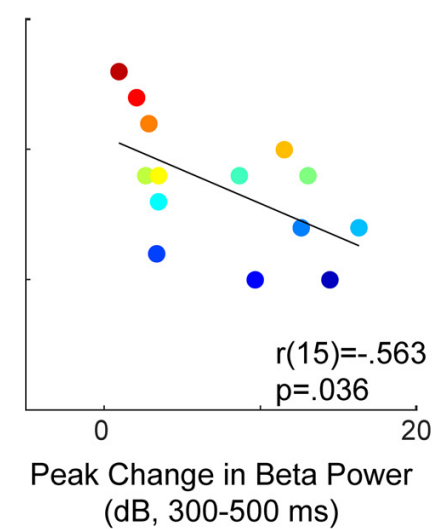

FIGURE 4 | Relationships between perturbation-evoked beta power (baseline subtracted) during overall (A), early (B), and later-phase balance reactions (C). No relationships were observed between perturbation-evoked beta power during overall (100-500 ms) or early-phase (100-300 ms), but later-phase (300-500 ms) perturbation-evoked beta power was negatively associated with miniBEST score. Individual color bar scaled by miniBEST score throughout (red = highest miniBEST, blue = lowest miniBEST).

\section{Prefrontal-Motor Cortical Network Engagement Mediates Cognitive Interference and Reactive Balance Capacity in Older Adults}

Prefrontal-motor circuit engagement may mediate cognitive dual-task interference and reactive balance capacity in older adults, as we found that individuals with greater perturbationevoked prefrontal-motor cortical coherence displayed greater slowing of dynamic balance performance during cognitive loading and took reactive steps at lower perturbation magnitudes (Figure 5). Our findings complement those of previous studies suggesting that over-recruitment of prefrontal cortical resources at low levels of motor difficulty imposes a ceiling effect on walking capacity in older adults when walking difficulty is increased with obstacle challenges, particularly after stroke (Clark et al., 2014; Hawkins et al., 2018). Our findings suggest the presence of a similar ceiling effect for prefrontal-motor network engagement in older adults during balance reactions at low levels of perturbation difficulty across participants. Our results further expand on these previous findings by identifying the motor circuit specificity of prefrontal networks and applying these circuit-specific mechanisms within the context of balance-correcting behavior. Here, older adults with greater interactions between prefrontal and motor cortical regions at a given low magnitude perturbation may have been closer to their "ceiling" for prefrontal-motor cortical engagement. An advantage of our experimental paradigm that measured cortical activity at the same perturbation magnitude across the entire participant cohort is that we were able to index cortical engagement of individuals who were each subjected to a different level of individual balance challenge according to their ability. As such, we demonstrated the effect of the balance challenge on differences in the level of cortical engagement across individuals. Individual differences in cortical engagement to the same perturbation were strongly associated with the individual-specific level of balance challenge posed by the perturbation, as assessed by the step threshold. There may also be other factors affecting the engagement of cortical resources in balance control; these may be more effectively identified by using a normalized level of balance challenge across individuals in future studies. Our data suggest that normalizing perturbation characteristics to step threshold may be one way to identify similar levels of balance challenge across individuals; we scaled acceleration, velocity, and displacement together, however, their individual effects on balance challenge need to be investigated further. Our findings also implicate that the individuals who had greater levels of prefrontal-motor circuit engagement at the same perturbation magnitude may have had reduced availability of cortical resources as balance perturbation magnitude was increased during the reactive step threshold assessment, ultimately necessitating a reactive stepping response at lower perturbation magnitudes. These findings identify prefrontal-motor cortical networks as a potential target for fall prevention strategies aimed specifically at raising the upper-end of individual reactive balance capacity and improving balance performance under cognitive loading conditions.

In the face of a destabilizing postural event, some older adults may capitalize on prefrontal-motor circuit engagement to maintain standing balance, a neural strategy which has distinct behavioral consequences. Perturbation-evoked prefrontal-motor network interactions may reflect executive function and working memory (Naghavi and Nyberg, 2005) and potentially interact as part of a cognitive predictive coding framework (Moran et al., 2014). This prefrontal-motor network engagement during balance reactions may effectively contribute to predictive performance in the aging brain, as it becomes increasingly more accurate in generating predictive models of the environment (Moran et al., 2014), possibly compensating for age-related declines in sensory processing and feedback mechanisms. 
A Prefrontal-Motor Coherence Examples

70 yo M, miniBEST 28, Step threshold: 18

Single TUG: 7.0s, Dual TUG: $7.8 \mathrm{~s}$

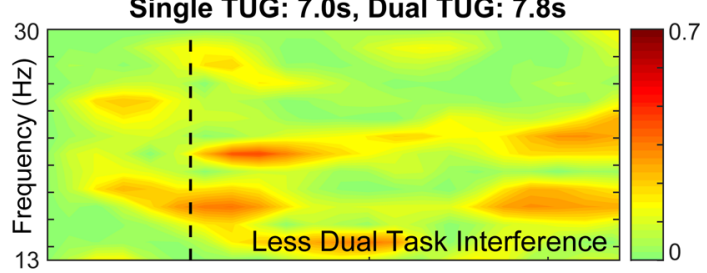

73 yo $M$, miniBEST 24, Step threshold: 7

Single TUG: 7.5s, Dual TUG: $10.2 \mathrm{~s}$
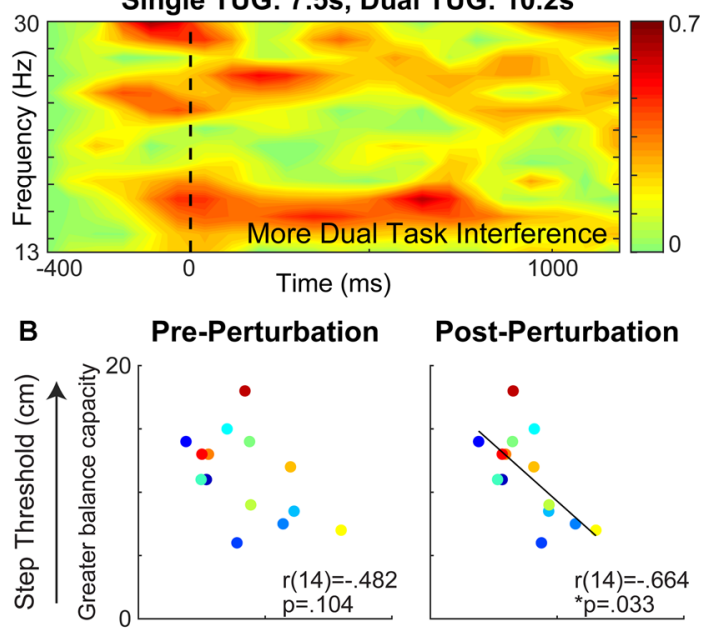

Post-Perturbation

C
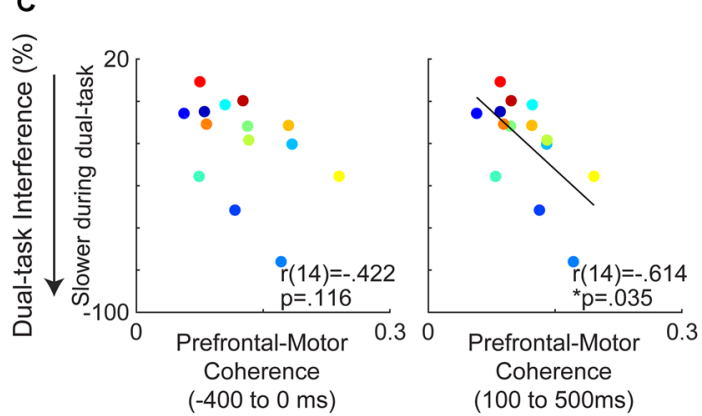

FIGURE 5 | Prefrontal-motor cortical beta coherence during balance reactions and relationships to balance behavior. Prefrontal-motor beta coherence was different between individuals with less vs. more cognitive dual-task interference. The broken line indicates the onset of perturbation (A) Prefrontal-motor coherence was negatively associated with reactive step threshold ( $p=0.033)$ (B) and cognitive dual-task interference $(p=0.035)$ (C) at post-perturbation but not pre-perturbation time-points.

Another possibility is that recruitment of prefrontal-motor circuits reflects a greater focus of attention directed towards balance control when the balance is challenged (Boisgontier and Nougier, 2013), though, unlike motor cortical beta power, it was not associated with general clinical balance ability measured in the miniBEST. In any case, the engagement of prefrontalmotor circuits during balance reactions may provide some older adults with an effective mechanism to control posture at lower perturbation difficulty levels, enabling them to achieve similar miniBEST scores to individuals who engage different neural control strategies. However, possibly as a result, older adults who rely on prefrontal-motor circuits for balance control tended to show more compromised balance performance when a cognitive demand was placed on the limited pool of executive resources during the cognitive dual-task performance (Figure 5). Our results may provide a foundation for future research testing the clinical utility of EEG measures of cortical activity during balance reactions as a more sensitive and specific assessment tool for balance control in the earliest preclinical stages of age-related balance impairments. As a first step, clinical balance testing under cognitive dual-task conditions may serve as an important adjunct in a battery of clinical balance assessments, as it elucidates the neural strategy that individuals use to support balance control and may predict balance performance under specific task conditions where attentional and executive control resources are concurrently loaded. Further, these findings suggest that rehabilitation interventions for fall prevention that yield little change in clinical miniBEST scores may not necessarily be ineffective. The induced clinical benefits of rehabilitation may come to light in improvements in balance safety, community function, and independence associated with concurrent mobility and cognitive function.

\section{Somatosensory-Motor Network Connectivity Is Not Associated With Clinical Balance Ability in Older Adults}

In the present study, it was surprising that functional connectivity of somatosensory-motor circuits was not associated with any balance behavioral metric in older adults. In contrast to beta power which appears to reflect overall upregulation of cortical excitability (Aono et al., 2013; Takemi et al., 2013a,b), beta coherence is thought to reflect interactive coupling between cortical regions (Nolte et al., 2004) necessary for information integration and processing (Nolte et al., 2004; Fries, 2005, 2015). Thus, the absence of a relationship between somatosensory-motor beta coherence, in contrast to the presence of a relationship with motor cortical beta power and miniBEST (Figure 4C), may be explained by different cortical mechanisms indexed by each metric contributing to different aspects of balance control.

The lack of an association between somatosensory-motor circuit connectivity during balance reactions and balance behavior in older adults may also implicate the role of other brain networks for sensory system integration for balance control, which may work to counteract age-related impairments in peripheral somatosensory system function, as previously postulated (Lenz et al., 2012; Clark, 2015; Pleger et al., 2016). Pervasive declines in sensorimotor processing with aging have been well-documented (Zhang et al., 2011) and may impair the function of the fast-acting subcortical brainstem and spinalmediated circuits for postural control (Baudry and Duchateau, 2012; Hortobágyi et al., 2018; Henry and Baudry, 2019), contributing to the prevalence of balance dysfunction in older adults (Hortobágyi et al., 2018; Henry and Baudry, 2019). However, the lack of relationship between somatosensorymotor coherence and balance behavior in the present study 
TABLE 2 | Statistical analyses for relationships between cortical beta coherence vs. balance behavior.

\begin{tabular}{|c|c|c|c|c|}
\hline \multirow[t]{2}{*}{ Balance behavior } & \multicolumn{2}{|c|}{ Somatosensory-motor coherence } & \multicolumn{2}{|c|}{ Prefrontal-motor coherence } \\
\hline & Pre & Post & Pre & Post \\
\hline $\operatorname{miniBEST}$ & $0.037(0.524)$ & $0.238(0.320)$ & $0.069(0.524)$ & $-0.057(0.524)$ \\
\hline Step Threshold & $-0.549(0.093)$ & $-0.431(0.116)$ & $-0.482(0.104)$ & $-0.664(0.033)^{\star}$ \\
\hline Dual-Task Interference & $-0.526(0.093)$ & $-0.471(0.104)$ & $-0.422(0.116)$ & $-0.614(0.035)^{\star}$ \\
\hline
\end{tabular}

Pearson product moment correlation coefficients (FDR-corrected $p$ value). *Significant $p<0.05$.

suggests that other sensory systems and brain regions could provide compensatory support for impaired somatosensory system function for balance and mobility with aging. As individuals encounter higher levels of balance challenge, the recruitment of additional cortical resources (e.g., prefrontalmotor circuits) and/or other sensory systems (e.g., vestibular and visual) may be necessary to optimize motor control and could potentially compensate for age-related deficits of any individual sensory system (Hairston et al., 2003). In support of this theory, when older adults utilized multiple sensory systems (e.g., somatosensory, visual, auditory) for manual reactive task performance, they showed faster response times; these performance gains were even greater than those observed in younger adults (Laurienti et al., 2006). Additionally, the contrasting findings between cortical beta power and coherence over somatosensory and motor brain regions in the present study build upon those of Malcolm et al. (2020), who found greater modulation of cortical beta activity during quiet static standing in older compared to younger adults as postural difficulty increased with narrowing base of support (Malcolm et al., 2020). Here, our results suggest findings by Malcolm et al. (2020) may reflect a more generalized signal for the demand for increased general cortical recruitment rather than sensorimotor processing and integration. However, we may have been unequipped to fully capture a relationship between somatosensory-motor coherence and age-related balance impairments, as clinical balance testing may be limited in sensitivity to detect subtle balance impairments, and most participants in the present study had relatively high clinical balance function (mean miniBEST score $=23.6 \pm 3.0 / 28$, Table 1 ) and many were not within the advanced end-stages of aging processes (mean age $68.9 \pm 8.4$ years). Future research involving a larger participant cohort including older adults with lower levels of clinical balance ability and individuals across the lifespan could shed light on whether this theory prevails in the context of balance and mobility function.

\section{Role of Cortical Inhibitory Processes and Response Inhibition in Balance Control With Aging}

Cortical beta coherence during balance reactions in older adults may reveal important information about the role of age-related cortical inhibitory processes (Peiffer et al., 2007; Zwergal et al., 2012). Movement-related beta oscillations have been associated with inhibitory GABAergic network activity (Baker, 2007; Muthukumaraswamy et al., 2013), which can precisely influence corticomotor output through synaptic connections with pyramidal neurons, fine-tuning motor control processes
(Yamawaki et al., 2008). Over the course of aging, there is a general decline in neural inhibitory processes (Heise et al., 2013; Papegaaij et al., 2014). Evidence from the present study is consistent with the notion that robust modulation of beta oscillatory activity likely reflects, at least in part, greater response inhibition required to maintain the "status quo" body posture (Androulidakis et al., 2006; see Engel and Fries, 2010; for review). In line with previous research showing reduced corticomuscular beta coherence when participants expected a probable (Schoffelen et al., 2005) or imminent motor event to occur Androulidakis et al. (2007), Solis-Escalante et al. (2019) found that the modulation of cortical beta activity during balance reactions with a feet-in-place strategy was attenuated when participants were instructed to execute a voluntary stepping response during the same perturbations. Maintenance of upright standing posture in the absence of a planned reactive step required greater motor control effort and was accompanied by a more robust modulation of beta power (Solis-Escalante et al., 2019). In response to an unexpected event, Wessel and Aron (2017) proposed the recruitment of a "global suppressive network;" in the context of standing balance reactions, this suggests that increased cortical beta power may index top-down corticomotor inhibition required to suppress the default stepping response strategy, requiring greater inhibition of this response during the most challenging balance conditions (Wessel and Aron, 2017; Wessel, 2020). As such, it is possible that individuals with the lowest general clinical balance ability and greatest motor cortical beta oscillatory power in response to the same perturbation magnitude demanded greater cortically-mediated response inhibition of undesired stepping responses in an attempt to maintain the pre-perturbation standing posture.

In particular, our findings support previous studies implicating a central role of frontal-motor networks in response inhibition, extending these findings to the engagement of cortical inhibitory mechanisms involving frontal cortical regions for motor suppression of unintentional stepping responses. The prefrontal cortex is a foundational node for the fronto-subthalamic-motor cortical inhibitory network that mediates response-conflict in the motor system (Wessel et al., 2019; Jana et al., 2020). If prefrontal-motor beta coherence reflects inhibitory mechanisms associated with this network, individuals with greater interactions between prefrontal-motor regions during balance reactions may have required greater effort to suppress stepping reactions in the present study, ultimately yielding lower reactive step thresholds in these older adults. As such, reactive step thresholds may provide a useful clinical probe for declines in inhibitory cortical network function in older adults. Using transcranial magnetic 
stimulation to directly perturb ongoing motor cortical activity, our lab previously found that event-related motor cortical beta coherence appears to reflect the ability to flexibly modulate cortical network interactions between states of rest and active lower limb muscle contraction; this motor cortical flexibility was linked to functional walking impairment in older adults after stroke (Palmer et al., 2020). While somatosensory-motor circuits may contribute to baseline standing postural control, our results suggest that the ability of the prefrontal-motor cortical circuits to flexibly engage in balance reactions during a destabilizing postural event may be crucial for upper-end reactive balance control. Here, lower perturbation-evoked prefrontal-motor cortical connectivity in older adults with lower stepping thresholds could stem from an age-related decline in inhibitory network function and consequently reduce the flexible modulatory capacity of the motor system to respond to destabilizing balance events. It remains unclear why some individuals may be more resistant to age-related declines in neural inhibitory processes. More research is needed to improve our understanding of the underlying causal factors for differences in cortical circuit engagement during reactive balance between individuals in aging populations. However, lifestyle behaviors, particularly physical activity, appear to play a key role in the slowing of normal age-related declines in inhibitory neural network function (Levin and Netz, 2015).

\section{LIMITATIONS}

Our electrode-based analysis approach limits the spatial anatomical specificity of the current findings; however, the advantage of the approach is to offer clinically-feasible biomarkers in older adults and patient populations. Future studies utilizing more sophisticated source localization approaches coupling dynamic EEG activity with structural MRI analyses will enhance the spatial resolution to more precisely localize cortical contributions to individual-specific neural network dynamics identified in the present study. Given the proximity of the channels overlying these cortical regions, we employed a conservative IPC approach for functional connectivity analyses that minimizes instantaneous common source activity, supported by the relatively low coherence values observed in the present study. Though this conservative approach risks underestimating the degree of functional connectivity relative to other approaches, it increases the confidence that coherence measures reflect a greater degree of true neural interactions between sources. A limitation of IPC analysis is that we cannot determine the directionality of these neural interactions. It is likely that somatosensory-motor and prefrontal-motor coherence measures here reflect both feedforward (e.g., somatosensory-to-motor and prefrontal-tomotor) and feedback (e.g., motor-to-somatosensory and motorto-prefrontal) network interactions. Future studies could assess the directionality of these interactions with effective connectivity approaches. During cognitive dual-task performance assessment, we quantified motor behavioral performance, but cognitive performance was not quantified. Future studies may address this limitation by quantifying concurrent declines in balance and cognitive performance that have been reported in older adults. The cortical event-related potentials evoked in response to standing balance perturbations show a similar pattern to those evoked by other tasks, for example, those involving cognitive error detection (see Payne et al., 2019b for a comprehensive review). Future studies employing an active control condition could help delineate whether perturbation-evoked measures of cortical activity are specific to reactive balance responses or may reflect common neural mechanisms for detecting errors.

\section{CONCLUSIONS}

Our findings show that older adults engage individual circuitspecific cortical strategies during balance behavior that are linked to distinct aspects of balance control. Our results suggest that prefrontally-mediated cortical strategies may be less effective for balance control in distracting contexts and under the most challenging balance conditions. The cortical strategy utilized by the individual may have unique contributions to balance behavior depending on the context (e.g., concurrent cognitive load) and/or the environment (e.g., level of balance difficulty). Our findings potentially identify suboptimal neural control strategies involving prefrontal-motor circuits that could be targeted for early intervention to prevent the emergence of clinical balance deficits in aging populations. Further, the novel findings of the present study provide effect sizes of cortical activity as a function of balance behavior which could serve as a foundation for larger mechanistic studies investigating the neural mechanisms of balance control in older adults (Julious, 2005). Finally, these findings motivate future studies to investigate whether similar cortical mechanisms are engaged during dynamic mobility behaviors involving balance destabilization as an important next step for the translation of these findings to clinical settings.

\section{DATA AVAILABILITY STATEMENT}

The raw data supporting the conclusions of this article will be made available by the authors, without undue reservation.

\section{ETHICS STATEMENT}

The studies involving human participants were reviewed and approved by Emory University Institutional Review Board. The patients/participants provided their written informed consent to participate in this study.

\section{AUTHOR CONTRIBUTIONS}

JP conceived the presented idea, designed the analysis for this study, and constructed the first draft of this manuscript. JP and AP collected the data and performed all data analyses. $\mathrm{AP}$ constructed the figures for the manuscript. AP and $\mathrm{MB}$ contributed the analysis tools for EEG data. MB and LT supervised the findings of this work. All authors contributed to the article and approved the submitted version. 


\section{FUNDING}

This work was supported by postdoctoral fellowship awards from the American Heart Association (AHA00035638) and the Eunice Kennedy Shriver National Institute of Child

\section{REFERENCES}

Alizadehsaravi, L., Bruijn, S. M., Maas, H., and van Dieën, J. H. (2020). Modulation of soleus muscle $\mathrm{H}$-reflexes and ankle muscle co-contraction with surface compliance during unipedal balancing in young and older adults. Exp. Brain Res. 238, 1371-1383. doi: 10.1007/s00221-020-05784-0

Androulidakis, A. G., Doyle, L. M. F., Gilbertson, T. P., and Brown, P. (2006). Corrective movements in response to displacements in visual feedback are more effective during periods of $13-35 \mathrm{~Hz}$ oscillatory synchrony in the human corticospinal system. Eur. J. Neurosci. 24, 3299-3304. doi: 10.1111/j.1460-9568. 2006.05201.x

Androulidakis, A. G., Doyle, L. M. F., Yarrow, K., Litvak, V., Gilbertson, T. P., and Brown, P. (2007). Anticipatory changes in beta synchrony in the human corticospinal system and associated improvements in task performance. Eur. J. Neurosci. 25, 3758-3765. doi: 10.1111/j.1460-9568.2007.05620.x

Aono, K., Miyashita, S., Fujiwara, Y., Kodama, M., Hanayama, K., Masakado, Y., et al. (2013). Relationship between event-related desynchronization and cortical excitability in healthy subjects and stroke patients. Tokai J. Exp. Clin. Med. 38, 123-128.

Baker, S. N. (2007). Oscillatory interactions between sensorimotor cortex and the periphery. Curr. Opin. Neurobiol. 17, 649-655. doi: 10.1016/j.conb.2008.01.007

Baudry, S., and Duchateau, J. (2012). Age-related influence of vision and proprioception on Ia presynaptic inhibition in soleus muscle during upright stance. J. Physiol. 590, 5541-5554. doi: 10.1113/jphysiol.2012.228932

Boisgontier, M. P., and Nougier, V. (2013). Ageing of internal models: from a continuous to an intermittent proprioceptive control of movement. Age 35, 1339-1355. doi: 10.1007/s11357-012-9436-4

Bootsma, J. M., Caljouw, S. R., Veldman, M. P., Maurits, N. M., Rothwell, J. C., and Hortobágyi, T. (2020). Failure to engage neural plasticity through practice of a high-difficulty task is accompanied by reduced motor skill retention in older adults. Neuroscience 451, 22-35. doi: 10.1016/j.neuroscience.2020.10.013

Brown, L. A., Shumway-cook, A., and Woollacott, M. H. (1999). Attentional demands and postural recovery: the effects of aging. J. Gerontol. 54, 165-171. doi: 10.1093/gerona/54.4.m165

Brown, P. (2007). Abnormal oscillatory synchronization in the motor system leads to impaired movement. Curr. Opin. Neurobiol. 17, 656-664. doi: 10.1016/j. conb.2007.12.001

Cassady, K., Ruitenberg, M. F. L., Reuter-Lorenz, P. A., Tommerdahl, M., and Seidler, R. D. (2020). Neural Dedifferentiation across the Lifespan in the Motor and Somatosensory Systems. Cereb. Cortex 30, 3704-3716. doi: 10.1093/cercor/bhz336

Chandler, J. M., Duncan, P. W., and Studenski, S. A. (1990). Balance performance on the postural stress test: comparison of young adults, healthy elderly and fallers. Phys. Ther. 70, 410-415. doi: 10.1093/ptj/70.7.410

Chang, C.-J., Yang, T.-F., Yang, S.-W., and Chern, J.-S. (2016). Cortical modulation of motor control biofeedback among the elderly with high fall risk during a posture perturbation task with augmented reality. Front. Aging Neurosci. 8:80. doi: 10.3389/fnagi.2016.00080

Chen, M., Pillemer, S., England, S., Izzetoglu, M., Mahoney, J. R., and Holtzer, R. (2017). Neural correlates of obstacle negotiation in older adults: an fNIRS study. Gait Posture 58, 130-135. doi: 10.1016/j.gaitpost.2017.07.043

Cid-Fernández, S., Lindín, M., and Díaz, F. (2014). Effects of aging and involuntary capture of attention on event-related potentials associated with the processing of and the response to a target stimulus. Front. Hum. Neurosci. 8:745. doi: 10.3389/fnhum.2014.00745

Clark, D. J. (2015). Automaticity of walking: functional significance, mechanisms, measurement and rehabilitation strategies. Front. Hum. Neurosci. 9:246. doi: 10.3389/fnhum.2015.00246
Health \& Human Development of the National Institutes of Health (F32HD096816) to JP, (K12HD055931) to MB, and (5T90DA032466, 1P50NS098685, R01 HD46922) to LT, and a grant from the National Science Foundation (1137229) to LT.

Clark, D. J., Manini, T. M., Ferris, D. P., Hass, C. J., Brumback, B. A., Cruz-Almeida, Y., et al. (2019). Multimodal imaging of brain activity to investigate walking and mobility decline in older adults (mind in motion study): hypothesis, theory and methods. Front. Aging Neurosci. 11:358. doi: 10.3389/fnagi.2019.00358

Clark, D. J., Rose, D. K., Ring, S. A., and Porges, E. C. (2014). Utilization of central nervous system resources for preparation and performance of complex walking tasks in older adults. Front. Aging Neurosci. 6:217. doi: 10.3389/fnagi. 2014.00217

Delorme, A., and Makeig, S. (2004). EEGLAB: an open source toolbox for analysis of single-trial EEG dynamics including independent component analysis. J. Neurosci. Methods 134, 9-21. doi: 10.1016/j.jneumeth.2003. 10.009

Engel, A. K., and Fries, P. (2010). Beta-band oscillations-signaling the status quo? Curr. Opin. Neurobiol. 20, 156-165. doi: 10.1016/j.conb.2010. 02.015

Fries, P. (2005). A mechanism for cognitive dynamics: neuronal communication through neuronal coherence. Trends Cogn. Sci. 9, 474-480. doi: 10.1016/j.tics. 2005.08.011

Fries, P. (2015). Rhythms for cognition: communication through coherence. Neuron 88, 220-235. doi: 10.1016/j.neuron.2015.09.034

Gagnon, H., Simmonite, M., Cassady, K., Chamberlain, J., Freiburger, E., Lalwani, P., et al. (2019). Michigan Neural Distinctiveness (MiND) study protocol: investigating the scope, causes and consequences of age-related neural dedifferentiation. BMC Neurol. 19:61. doi: 10.1186/s12883-0191294-6

Ghosn, N. J., Palmer, J. A., Borich, M. R., Ting, L. H., and Payne, A. M. (2020). Cortical beta oscillatory activity evoked during reactive balance recovery scales with perturbation difficulty and individual balance ability. Brain Sci. 10:860. doi: 10.3390/brainsci10110860

Goble, D. J., Coxon, J. P., Van Impe, A., Geurts, M., Van Hecke, W., Sunaert, S., et al. (2012). The neural basis of central proprioceptive processing in older versus younger adults: An important sensory role for right putamen. Hum. Brain Mapp. 33, 895-908. doi: 10.1002/hbm.21257

Grefkes, C., Nowak, D. A., Eickhoff, S. B., Dafotakis, M., Küst, J., Karbe, H., et al. (2008). Cortical connectivity after subcortical stroke assessed with functional magnetic resonance imaging. Ann. Neurol. 63, 236-246. doi: 10.1002/ana. 21228

Guadagnoli, M. A., and Lee, T. D. (2004). Challenge point: a framework for conceptualizing the effects of various practice conditions in motor learning. J. Mot. Behav. 36, 212-224. doi: 10.3200/JMBR.36.2.212-224

Hairston, W. D., Laurienti, P. J., Mishra, G., Burdette, J. H., and Wallace, M. T. (2003). Multisensory enhancement of localization under conditions of induced myopia. Exp. Brain Res. 152, 404-408. doi: 10.1007/s00221-0031646-7

Hawkins, K. A., Fox, E. J., Daly, J. J., Rose, D. K., Christou, E. A., McGuirk, T. E., et al. (2018). Prefrontal over-activation during walking in people with mobility deficits: interpretation and functional implications. Hum. Mov. Sci. 59, 46-55. doi: 10.1016/j.humov.2018.03.010

Heise, K.-F., Zimerman, M., Hoppe, J., Gerloff, C., Wegscheider, K., and Hummel, F. C. (2013). The aging motor system as a model for plastic changes of gaba-mediated intracortical inhibition and their behavioral relevance. J. Neurosci. 33, 9039-9049. doi: 10.1523/JNEUROSCI.4094-12.2013

Henry, M., and Baudry, S. (2019). Age-related changes in leg proprioception: implications for postural control. J. Neurophysiol. 122, 525-538. doi: 10.1152/jn.00067.2019

Heuninckx, S., Wenderoth, N., and Swinnen, S. P. (2008). Systems neuroplasticity in the aging brain: recruiting additional neural resources 
for successful motor performance in elderly persons. J. Neurosci. 28, 91-99. doi: 10.1523/JNEUROSCI.3300-07.2008

Hortobágyi, T., van de Waardt, L. E., Tokuno, C. D., Taube, W., and Papegaaij, S. (2018). Age-related reversal of spinal excitability during anticipatory postural control. Eur. J. Appl. Physiol. 118, 2577-2585. doi: 10.1007/s00421-0183982-0

Jacobs, J. V., and Horak, F. B. (2007). Cortical control of postural responses. J. Neural Transm. 114, 1339-1348. doi: 10.1007/s00702-007-0657-0

Jana, S., Hannah, R., Muralidharan, V., and Aron, A. R. (2020). Temporal cascade of frontal, motor and muscle processes underlying human action-stopping. eLife 9:e50371. doi: 10.7554/eLife.50371

Jensen, J. L., Brown, L. A., and Woollacott, M. H. (2001). Compensatory stepping: the biomechanics of a preferred response among older adults. Exp. Aging Res. 27, 361-376. doi: 10.1080/03610730109342354

Johari, K., and Behroozmand, R. (2020). Event-related desynchronization of alpha and beta band neural oscillations predicts speech and limb motor timing deficits in normal aging. Behav. Brain Res. 393:112763. doi: 10.1016/j.bbr.2020. 112763

Julious, S. A. (2005). Sample size of 12 per group rule of thumb for a pilot study. Pharmaceut. Statist. 4, 287-291. doi: 10.1002/pst.185

Kim, K.-M., Hart, J. M., and Hertel, J. (2013). Influence of body position on fibularis longus and soleus Hoffmann reflexes. Gait Posture 37, 138-140. doi: 10.1016/j.gaitpost.2012.06.009

Langan, J., Peltier, S. J., Bo, J., Fling, B. W., Welsh, R. C., and Seidler, R. D. (2010). Functional implications of age differences in motor system connectivity. Front. Sys. Neurosci. 4:17. doi: 10.3389/fnsys.2010.00017

Laurienti, P. J., Burdette, J. H., Maldjian, J. A., and Wallace, M. T. (2006). Enhanced multisensory integration in older adults. Neurobiol. Aging 27, 1155-1163. doi: 10.1016/j.neurobiolaging.2005.05.024

Lenz, M., Tegenthoff, M., Kohlhaas, K., Stude, P., Höffken, O., Gatica Tossi, M. A., et al. (2012). Increased excitability of somatosensory cortex in aged humans is associated with impaired tactile acuity. J. Neurosci. 32, 1811-1816. doi: 10.1523/JNEUROSCI.2722-11.2012

Leone, C., Feys, P., Moumdjian, L., D'Amico, E., Zappia, M., and Patti, F. (2017). Cognitive-motor dual-task interference: a systematic review of neural correlates. Neurosci. Biobehav. Rev. 75, 348-360. doi: 10.1016/j.neubiorev.2017. 01.010

Levin, O., and Netz, Y. (2015). Aerobic training as a means to enhance inhibition: what's yet to be studied? Eur. Rev. Aging Phys. Activ. 12:14. doi: 10.1186/s11556015-0160-9

Li, W., Keegan, T. H. M., Sternfeld, B., Sidney, S., Quesenberry, C. P., and Kelsey, J. L. (2006). Outdoor falls among middle-aged and older adults: a neglected public health problem. Am. J. Public Health 96, 1192-1200. doi: 10.2105/AJPH.2005.083055

Lundin-Olsson, L., Nyberg, L., and Gustafson, Y. (1997). "Stops walking when talking" as a predictor of falls in elderly people. Lancet 349:617. doi: 10.1016/S0140-6736(97)24009-2

Makeig, S. (1993). Auditory event-related dynamics of the EEG spectrum and effects of exposure to tones. Electroencephalogr. Clin. Neurophysiol. 86, 283-293. doi: 10.1016/0013-4694(93)90110-h

Makeig, S., Delorme, A., Westerfield, M., Jung, T.-P., Townsend, J., Courchesne, E., et al. (2004). Electroencephalographic brain dynamics following manually responded visual targets. PLoS Biol. 2:e176. doi: 10.1371/journal.pbio.0020176

Maki, B. E., and McIlroy, W. E. (1996). Postural control in the older adult. Clin. Geriatr. Med. 12, 635-658.

Maki, B. E., and McIlroy, W. E. (2006). Control of rapid limb movements for balance recovery: age-related changes and implications for fall prevention. Age Ageing 35, ii12-ii18. doi: 10.1093/ageing/afl078

Maki, B. E., and McIlroy, W. E. (2007). Cognitive demands and cortical control of human balance-recovery reactions. J. Neural Transm. 114, 1279-1296. doi: 10.1007/s00702-007-0764-y

Malcolm, B. R., Foxe, J. J., Joshi, S., Verghese, J., Mahoney, J. R., Molholm, S., et al. (2020). Aging-related changes in cortical mechanisms supporting postural control during base of support and optic flow manipulations. Eur. J. Neurosci. 1-19. doi: 10.1111/ejn.15004

Marques, A., Almeida, S., Carvalho, J., Cruz, J., Oliveira, A., and Jácome, C. (2016). Reliability, validity and ability to identify fall status of the balance evaluation systems test, mini-balance evaluation systems test and brief-balance evaluation systems test in older people living in the community. Arch. Phys. Med. Rehabil. 97, 2166-2173.e1. doi: 10.1016/j.apmr.2016.07.011

Mattay, V. S., Fera, F., Tessitore, A., Hariri, A. R., Das, S., Callicott, J. H., et al. (2002). Neurophysiological correlates of age-related changes in human motor function. Neurology 58, 630-635. doi: 10.1212/wnl.58.4.630

McIlroy, W. E., and Maki, B. E. (1994). The "deceleration response" to transient perturbation of upright stance. Neurosci. Lett. 175, 13-16. doi: 10.1016/03043940(94)91066-9

Mille, M.-L., Rogers, M. W., Martinez, K., Hedman, L. D., Johnson, M. E., Lord, S. R., et al. (2003). Thresholds for inducing protective stepping responses to external perturbations of human standing. J. Neurophysiol. 90, 666-674. doi: $10.1152 /$ jn.00974.2002

Mirelman, A., Maidan, I., Bernad-Elazari, H., Shustack, S., Giladi, N., and Hausdorff, J. M. (2017). Effects of aging on prefrontal brain activation during challenging walking conditions. Brain Cogn. 115, 41-46. doi: 10.1016/j.bandc. 2017.04.002

Montero-Odasso, M., Verghese, J., Beauchet, O., and Hausdorff, J. M. (2012). Gait and cognition: a complementary approach to understanding brain function and the risk of falling. J. Am. Geriatr. Soc. 60, 2127-2136. doi: 10.1111/j.1532-5415. 2012.04209.x

Moran, R. J., Symmonds, M., Dolan, R. J., and Friston, K. J. (2014). The brain ages optimally to model its environment: evidence from sensory learning over the adult lifespan. PLoS Comput. Biol. 10:e1003422. doi: 10.1371/journal.pcbi. 1003422

Morris, R., Lord, S., Bunce, J., Burn, D., and Rochester, L. (2016). Gait and cognition: Mapping the global and discrete relationships in ageing and neurodegenerative disease. Neurosci. Biobehav. Rev. 64, 326-345. doi: 10.1016/j. neubiorev.2016.02.012

Muthukumaraswamy, S. D., Myers, J. F. M., Wilson, S. J., Nutt, D. J., LingfordHughes, A., Singh, K. D., et al. (2013). The effects of elevated endogenous GABA levels on movement-related network oscillations. NeuroImage 66, 36-41. doi: 10.1016/j.neuroimage.2012.10.054

Naghavi, H. R., and Nyberg, L. (2005). Common fronto-parietal activity in attention, memory and consciousness: shared demands on integration? Conscious Cogn. 14, 390-425. doi: 10.1016/j.concog.2004.10.003

Nolte, G., Bai, O., Wheaton, L., Mari, Z., Vorbach, S., and Hallett, M. (2004). Identifying true brain interaction from EEG data using the imaginary part of coherency. Clin. Neurophysiol. 115, 2292-2307. doi: 10.1016/j.clinph.2004. 04.029

Palmer, J. A., Kesar, T. M., Wolf, S. L., and Borich, M. R. (2020). Cortical motor network flexibility during lower limb motor activity and deficiencies after stroke. medRxiv [Preprint]. doi: 10.1101/2020.06.15.201 30773

Papegaaij, S., Taube, W., Baudry, S., Otten, E., and Hortobágyi, T. (2014). Aging causes a reorganization of cortical and spinal control of posture. Front. Aging Neurosci. 6, 1-15. doi: 10.3389/fnagi.2014.00028

Payer, D., Marshuetz, C., Sutton, B., Hebrank, A., Welsh, R. C., and Park, D. C. (2006). Decreased neural specialization in old adults on a working memory task. NeuroReport 17, 487-491. doi: 10.1097/01.wnr.0000209005.40 481.31

Payne, A. M., and Ting, L. H. (2020). Worse balance is associated with larger perturbation-evoked cortical responses in healthy young adults. Gait Posture 80, 324-330. doi: 10.1016/j.gaitpost.2020.06.018

Payne, A. M., Hajcak, G., and Ting, L. H. (2019a). Dissociation of muscle and cortical response scaling to balance perturbation acceleration. J. Neurophysiol. 121, 867-880. doi: 10.1152/jn.00237.2018

Payne, A. M., Ting, L. H., and Hajcak, G. (2019b). Do sensorimotor perturbations to standing balance elicit an error-related negativity? Psychophysiology 56, 1-12. doi: 10.1111/psyp.13359

Peiffer, A. M., Hugenschmidt, C. E., Maldjian, J. A., Casanova, R., Srikanth, R., Hayasaka, S., et al. (2007). Aging and the interaction of sensory cortical function and structure. Hum. Brain Mapp. 30, 228-240. doi: 10.1002/hbm. 20497

Pleger, B., Wilimzig, C., Nicolas, V., Kalisch, T., Ragert, P., Tegenthoff, M., et al. (2016). A complementary role of intracortical inhibition in age-related tactile degradation and its remodeling in humans. Sci. Rep. 6:27388 doi: $10.1038 /$ srep27388 
Plummer, P., and Eskes, G. (2015). Measuring treatment effects on dual-task performance: a framework for research and clinical practice. Front. Hum. Neurosci. 9:225. doi: 10.3389/fnhum.2015.00225

Rankin, J. K., Woollacott, M. H., Shumway-cook, A., and Brown, L. A. (2000). Cognitive influence on postural stability: a neuromuscular analysis in young and older adults. J. Gerontol. A Biol. Sci. Med. Sci. 55, 112-119. doi: 10.1093/gerona/55.3.m112

Ren, J., Wu, Y. D., Chan, J. S., and Yan, J. H. (2013). Cognitive aging affects motor performance and learning. Geriatr. Gerontol. Int. 13, 19-27. doi: 10.1111/j. 1447-0594.2012.00914.x

Robinovitch, S. N., Feldman, F., Yang, Y., Schonnop, R., Leung, P. M., Sarraf, T., et al. (2013). Video capture of the circumstances of falls in elderly people residing in long-term care: an observational study. Lancet 381, 47-54. doi: 10.1016/S0140-6736(12)61263-X

Rogasch, N. C., Sullivan, C., Thomson, R. H., Rose, N. S., Bailey, N. W., Fitzgerald, P. B., et al. (2017). Analysing concurrent transcranial magnetic stimulation and electroencephalographic data: a review and introduction to the open-source TESA software. NeuroImage 147, 934-951. doi: 10.1016/j. neuroimage.2016.10.031

Rossiter, H. E., Davis, E. M., Clark, E. V., Boudrias, M., and Ward, N. S. (2014). Beta oscillations reflect changes in motor cortex inhibition in healthy ageing. NeuroImage 91, 360-365. doi: 10.1016/j.neuroimage.2014.01.012

Rowe, J., Stephan, K. E., Friston, K., Frackowiak, R., Lees, A., and Passingham, R. (2002). Attention to action in Parkinson's disease: impaired effective connectivity among frontal cortical regions. Brain 125, 276-289. doi: 10.1093/brain/awf036

Schoffelen, J.-M., Oostenveld, R., and Fries, P. (2005). Neuronal coherence as a mechanism of effective corticospinal interaction. Science 308, 111-113. doi: 10.1126/science.1107027

Seidler, R. D., Bernard, J. A., Burutolu, T. B., Fling, B. W., Gordon, M. T., Gwin, J. T., et al. (2010). Motor control and aging: links to age-related brain structural, functional and biochemical effects. Neurosci. Biobehav. Rev. 34, 721-733. doi: 10.1016/j.neubiorev.2009.10.005

Seidler, R., Erdeniz, B., Koppelmans, V., Hirsiger, S., Mérillat, S., and Jäncke, L. (2015). Associations between age, motor function and resting state sensorimotor network connectivity in healthy older adults. Neuroimage 108, 47-59. doi: 10.1016/j.neuroimage.2014.12.023

Shumway-Cook, A., Woollacott, M., Kerns, K. A., and Baldwin, M. (1997). The effects of two types of cognitive tasks on postural stability in older adults with and without a history of falls. J. Gerontol. A Biol. Sci. Med. Sci. 52, M232-M240. doi: 10.1093/gerona/52a.4. $\mathrm{m} 232$

Solesio-Jofre, E., Serbruyns, L., Woolley, D. G., Mantini, D., Beets, I. A. M., and Swinnen, S. P. (2014). Aging effects on the resting state motor network and interlimb coordination. Hum. Brain Mapp. 35, 3945-3961. doi: 10.1002/hbm. 22450

Solis-Escalante, T., van der Cruijsen, J., de Kam, D., van Kordelaar, J., Weerdesteyn, V., and Schouten, A. C. (2019). Cortical dynamics during preparation and execution of reactive balance responses with distinct postural demands. NeuroImage 188, 557-571. doi: 10.1016/j.neuroimage.2018.12.045

Takemi, M., Masakado, Y., Liu, M., and Ushiba, J. (2013a). Event-related desynchronization reflects downregulation of intracortical inhibition in human primary motor cortex. J. Neurophysiol. 110, 1158-1166. doi: 10.1152/jn.01092. 2012

Takemi, M., Masakado, Y., Liu, M., and Ushiba, J. (2013b). Is event-related desynchronization a biomarker representing corticospinal excitability? Annu. Int. Conf. IEEE Eng. Med. Biol. Soc. 2013, 281-284. doi: 10.1109/EMBC.2013. 6609492

Tang, P.-F., Yang, H.-J., Peng, Y.-C., and Chen, H.-Y. (2015). Motor dual-task Timed Up \& go test better identifies prefrailty individuals than single-task timed up \& go test. Geriatr. Gerontol. Int. 15, 204-210. doi: 10.1111/ggi.12258

van Wijk, B. C. M., Beek, P. J., and Daffertshofer, A. (2012). Neural synchrony within the motor system: what have we learned so far? Front. Hum. Neurosci. 6:252. doi: 10.3389/fnhum.2012.00252

Wessel, J. R. (2020). $\beta$-bursts reveal the trial-to-trial dynamics of movement initiation and cancellation. J. Neurosci. 40, 411-423. doi: 10.1523/JNEUROSCI. 1887-19.2019

Wessel, J. R., and Aron, A. R. (2017). On the globality of motor suppression: unexpected events and their influence on behavior and cognition. Neuron 93 , 259-280. doi: 10.1016/j.neuron.2016.12.013

Wessel, J. R., Waller, D. A., and Greenlee, J. D. (2019). Non-selective inhibition of inappropriate motor-tendencies during response-conflict by a frontosubthalamic mechanism. eLife 8:e42959. doi: 10.7554/eLife.42959

Wolfson, L. I., Whipple, R., Amerman, P., and Kleinberg, A. (1986). Stressing the postural response. A quantitative method for testing balance. J. Am. Geriatr. Soc. 34, 845-850. doi: 10.1111/j.1532-5415.1986.tb07256.x

Woollacott, M., and Shumway-Cook, A. (2002). Attention and the control of posture and gait: a review of an emerging area of research. Gait Posture 16, 1-14. doi: 10.1016/s0966-6362(01)00156-4

Yamawaki, N., Stanford, I. M., Hall, S. D., and Woodhall, G. L. (2008). Pharmacologically induced and stimulus evoked rhythmic neuronal oscillatory activity in the primary motor cortex in vitro. Neuroscience 151, 386-395. doi: 10.1016/j.neuroscience.2007.10.021

Zaepffel, M., Trachel, R., Kilavik, B. E., and Brochier, T. (2013). Modulations of EEG beta power during planning and execution of grasping movements. PLoS One 8, 1-10. doi: 10.1371/journal.pone.0060060

Zhang, Z., Francisco, E. M., Holden, J. K., Dennis, R. G., and Tommerdahl, M. (2011). Somatosensory information processing in the aging population. Front. Aging Neurosci. 3:18. doi: 10.3389/fnagi.2011.00018

Zwergal, A., Linn, J., Xiong, G., Brandt, T. , Strupp, M., Jahn, K., et al. (2012). Aging of human supraspinal locomotor and postural control in fMRI. Neurobiol. Aging 33, 1073-1084. doi: 10.1016/j.neurobiolaging.2010.09.022

Conflict of Interest: The authors declare that the research was conducted in the absence of any commercial or financial relationships that could be construed as a potential conflict of interest.

Copyright (c) 2021 Palmer, Payne, Ting and Borich. This is an open-access article distributed under the terms of the Creative Commons Attribution License (CC BY). The use, distribution or reproduction in other forums is permitted, provided the original author(s) and the copyright owner(s) are credited and that the original publication in this journal is cited, in accordance with accepted academic practice. No use, distribution or reproduction is permitted which does not comply with these terms. 